\title{
Nitrofuranos y cloranfenicol, sustancias prohibidas para su uso en animales, presentes en alimentos agropecuarios primarios en el Perú (2011 - 2018)
}

\author{
Germán H. Correa-Núñez \\ Universidad Nacional Amazónica de Madre de Dios (Perú) \\ gcorrea@unamad.edu.pe \\ ORCID: https://orcid.org/0000-0002-4896-8120 \\ Jesús Eduardo Rojas Jaimes \\ Universidad Privada del Norte (Perú) \\ jesus.rojas.jaimes@gmail.com \\ ORCID: https://orcid.org/0000-0002-6910-9341 \\ Edward Morgan \\ Universidad Privada del Norte (Perú) \\ morgan0173028@gmail.com \\ ORCID: https://orcid.org/0000-0002-0558-0492 \\ Erick Chate Pérez \\ Universidad Privada del Norte (Perú) \\ goleadorerick23@gmail.com \\ ORCID: https://orcid.org/0000-0002-5552-8350
}

\section{RESUMEN}

El objetivo del trabajo fue revisar y analizar los resultados del monitoreo de los antibióticos prohibidos en producción animal para consumo humano, efectuados por el Servicio Nacional de Sanidad Agraria del Perú (SENASA): nitrofuranos (furaltadona y furazolidona) y cloranfenicol. Se efectuó análisis documental de los reportes de monitoreo de residuos químicos y otros contaminantes en alimentos agropecuarios primarios entre los años 2011 a 2018, disponibles en el sitio web del SENASA. Se concluye que tales xenobióticos han seguido usándose más allá del período de su prohibición y en algunas regiones su uso ha sido más reciente y más frecuente (a través de los años) y en más matrices alimentarias, por lo que se corresponde continuar con el monitoreo y a la vez cuantificar los hallazgos encontrados a efectos de prevenir potenciales efectos para la salud humana.

Palabras clave: cloranfenicol; nitrofuranos; producción animal; contaminantes alimentarios 


\title{
Nitrofurans and chloramphenicol, substances prohibited for use in
} animals, present in primary agricultural foods in Peru (2011 - 2018)

\begin{abstract}
The goal of this study was to review and analyze the results of the monitoring of antibiotics prohibited in animal production for human consumption, carried out by the National Agrarian Health Service of Peru (SENASA): nitrofurans (furaltadone and furazolidone) and chloramphenicol. A documentary analysis of the monitoring reports of chemical residues and other pollutants in primary agricultural foods was carried out between the years 2011 to 2018, available on the SENASA website. It is concluded that such xenobiotics have continued to be used beyond the period of their prohibition and in some regions their use has been more recent and more frequent (over the years) and in more food matrices, so it is appropriate to continue monitoring. and at the same time quantify the findings in order to prevent potential effects on human health.
\end{abstract}

Keywords: chloramphenicol; nitrofurans; animal production; food contaminants

Artículo recibido: 25 marzo 2021 Aceptado para publicación: 29 abril 2021 Correspondencia: gcorrea@unamad.edu.pe Conflictos de Interés: Ninguna que declarar 


\section{INTRODUCCIÓN}

En un mundo cada vez con mayor demanda de alimentos, la crianza intensiva de animales para la alimentación humana, así como la extracción acentuada de recursos naturales no renovables, aunado a una inadecuada recolección y disposición final de residuos sólidos no biodegradables, han conllevado a que las matrices suelo, aire y agua, así como los alimentos de consumo humano, contengan, cada vez más, diversos xenobióticos que pueden causar un estado de disbiosis perjudicial para la microbiota y salud del consumidor (Abdelsalam et., 2020), particularmente cuando se trata de presencia de metales pesados y residuos de antibióticos en productos cárnicos.

Los antibióticos son compuestos naturales, semisintéticos o sintéticos, utilizados contra infecciones bacterianas, ya sea eliminándolas (antibióticos strictu senso o bactericidas) o inhibiendo su crecimiento (bacteriostáticos). Ya en el siglo pasado, desde la cuarta década los antibióticos se han venido utilizando en la producción intensiva de animales de granja tales como cerdos, aves de corral y bovinos ya sea para prevenir la propagación de alguna enfermedad o para tratar a los animales infectados. De otro lado, también se viene aplicando bajas dosis de antibióticos a los piensos animales para promover su crecimiento y consecuentemente lograr una mayor producción de leche o carne en períodos reducidos de tiempo (Boyd, 2001). Adicionalmente, cuando se usa los antibióticos en bajas dosis se puede lograr una reducción en la mortalidad animal y una mejora en la tasa reproductiva, de ahí que su uso se haya extendido a nivel mundial. Ya para el año 2011, el 80\% de la venta de antibióticos en los Estados Unidos fue para su uso en animales productores de alimentos para consumo humano (FDA, 2013).

La demanda creciente de alimentos para atender a una población cada vez mayor ha conllevado a una crianza intensiva de animales para consumo humano, lo que a su vez acarrea la dosificación de los mismos con antibióticos para atenuar o controlar eventuales pérdidas atribuidas a enfermedades. Sin embargo, el residuo de estos fármacos puede permanecer en diferentes matrices (carne, leche, huevos, constituyendo un serio peligro para los consumidores. A causa de las propiedades de estos medicamentos, así como su perfil fisicoquímico, farmacocinético y farmacodinámico, e inapropiado o ausente período de ventana, estos residuos pueden desarrollar resistencia a antimicrobianos, causar reacciones anafilácticas, ser teratogénicos o mutagénicos, o provocar alteración de la microbiota intestinal (Beyene \& Assefa, 2015). Es precisamente el riesgo de contraer 
bacterias multirresistentes a antibióticos, conocidas como "superbugs", lo que hace relevante el consumo de alimentos y productos cárnicos con residuos de antibióticos (prohibidos o no), por la eventual transferencia de resistencia a antibióticos de animales a humanos, con efectos nocivos en la salud humana (Colombo \& Papetti, 2019).

En Estados Unidos, por ejemplo, rige un mecanismo estricto para ensayar todos los productos cárnicos, leche, huevos y aves de corral para detectar compuestos no deseados, entre los que se incluyen los residuos de antibióticos, tal como figura en el Programa Nacional de Residuos para producto cárnicos, de aves de corral y huevos - Planes de muestreo de residuos (USDA, 2015). En el Perú, el año 2013, mediante Resolución Directoral N. ${ }^{\circ}$ 0072-2013-MINAGRI-SENASA-DIAIA “se prohíbe la importación y comercialización de diversos principios activos, así como el uso de los mismos en la fabricación de productos veterinarios o alimentos para animales destinados al consumo humano y establecen otras disposiciones" entre ellos se cita a "el cloranfenicol es un cancerígeno y existen evidencias de que tiene un mecanismo genotóxico; los nitrofuranos, la furazolidona y nitrofurazona muestran evidencia de carcinogenicidad, con evidencias de un mecanismo genotóxico en el furazolidone, y la tumorigenicidad por un mecanismo indeterminado que afecta a los órganos de respuesta endocrina en el nitrofural (nitrofurazona)" (SENASA, 2013).

De otro lado, con la promulgación de la Resolución Ministerial N. ${ }^{\text {o } 372-2016 / M I N S A ~}$ del 03 de junio de 2016, se aprueba la NTS N. ${ }^{\circ}$ 120-MINSA/DIGESA-V.01 "Norma sanitaria que establece los límites máximos de residuos (LMR) de medicamentos veterinarios en alimentos de consumo humano" para fines de vigilancia y control sanitario en conformidad con el Decreto Legislativo N. ${ }^{\circ}$ 1062, Ley de inocuidad de los alimentos; encargando a la Dirección General de Salud Ambiental e Inocuidad Alimentaria, la difusión, implementación y supervisión de la referida resolución ministerial (MINSA, 2016) para lo cual se ha tomado como referencia los LMR establecidos en el Codex Alimentarius que constituye la norma internacional de referencia adoptada por el Perú en su normatividad nacional.

En los informes de Monitoreo de residuos químicos y otros contaminantes en alimentos agropecuarios primarios, del SENASA, entre los años 2011 a 2018 se considera a una muestra no conforme a "aquella muestra que contiene, como resultado del análisis, algún residuo de ingrediente activo de plaguicida químico de uso agrícola o medicamento 
veterinario en usos no registrados por la Autoridad Nacional o contiene una cantidad de residuos de sustancia activa mayor o no tiene límite máximo de residuos (LMR) establecido por el Codex Alimentarius" (SENASA, 2017). En consecuencia en la presente revisión se hace referencia a muestras no conformes.

En el Perú, desde el año 2011 al año 2018 el Servicio Nacional de Sanidad Agraria, a través de la Dirección de Insumos Agropecuarios e Inocuidad Agroalimentaria, ha venido publicando el Informe del monitoreo de contaminantes químicos en alimentos agropecuarios primarios, es decir, aquellos "alimentos agropecuarios de producción y de procesamiento primario destinados para el consumo humano"; donde, en algunos casos, se ha cuantificado el tipo de antibiótico detectado en la matriz alimentos para consumo humano. Sin embargo, a partir del año 2014 al año 2018, fecha del último informe, ya no se muestra información sobre la cuantificación del xenobiótico detectado. Para la determinación de residuos de medicamentos de uso veterinario el método utilizado fue cromatografía líquida acoplada a espectrometría de masa en tándem (LC/MS/MS) (SENASA, 2020).

El presente artículo tiene como objetivo efectuar una revisión crítica de los reportes de monitoreo de cloranfenicol y metabolitos de los nitrofuranos furaltadona y furazolidona, en alimentos agropecuarios primarios tomando como fue los reportes del monitoreo publicados en el website oficial del SENASA, entre el período 2011 a 2018, teniendo en cuenta la prohibición de su uso en el Perú desde el año 2013, por cuanto además no se dispone de LMR "Concentración máxima de residuos resultante del uso de un medicamento veterinario (expresada en $\mathrm{mg} / \mathrm{kg}$ o $\mu \mathrm{g} / \mathrm{kg}$ sobre la base del peso fresco) que la Comisión del Codex Alimentarius recomienda se permita legalmente o se reconozca como admisible dentro de un alimento o en la superficie del mismo" (SENASA, 2017; FAO-OMS, 2018).

\section{ESTRATEGIAS METODOLÓGICAS O MATERIALES Y MÉTODOS}

Se obtuvo información secundaria del sitio web oficial del Servicio Nacional de Sanidad Agraria del Perú, SENASA (https://www.senasa.gob.pe/senasa/programa-nacional-demonitoreo/) correspondiente al Programa Nacional de Monitoreo de Contaminantes en 10 ciudades, capitales de región, del Perú, la misma que se ha ordenado cronológicamente por año (2011 al 2018) para su respectiva interpretación y análisis, tabulando según el tipo de alimento agropecuario primario (matriz animal), antibiótico: cloranfenicol (CAP) 
o metabolito de antibiótico nitrofurano (AMOZ, para la furaltadona, y AOZ, para la furazolidona), región del país, y año de reporte, a nivel de establecimiento de producción y procesamiento primario, y de centros de expendio de estos productos.

\section{RESULTADOS Y DISCUSIÓN}

(se muestran en la siguiente página)

Tabla 1: Presencia de residuos de antibióticos en alimentos de procesamiento primario (2011-2016) 
Nitrofuranos y cloranfenicol

\begin{tabular}{|c|c|c|c|}
\hline Alimento agropecuario primario & Furaltadona (AMOZ) & Furazolidona (AOZ) & Cloranfenicol (CAP) \\
\hline \multicolumn{4}{|c|}{ Año 2011} \\
\hline Carne de ave (pollo) & Lima y Lambayeque & Lima y Lambayeque & 0 \\
\hline Carne de bovino & 0 & 0 & 0 \\
\hline Carne de ovino & 0 & 0 & 0 \\
\hline Carne de caprino & 0 & 0 & 0 \\
\hline Carne de porcino & Tacna y Cajamarca & Cajamarca y Tacna & 0 \\
\hline \multicolumn{4}{|c|}{ Año 2012} \\
\hline Carne de ave (pollo) & 0 & San Mart h y Tacna & 0 \\
\hline Carne de bovino & 0 & 0 & 0 \\
\hline Carne de ovino & La Libertad & La Libertad & Puno \\
\hline Carne de caprino & 0 & 0 & Ica \\
\hline Carne de porcino & 0 & Tacna y Piura & Ica \\
\hline Carne de cuy & 0 & 0 & 0 \\
\hline Leche cruda de bovino & 0 & 0 & 0 \\
\hline Alpaca/llama & 0 & 0 & 0 \\
\hline Miel de abeja & 0 & 0 & Tacna \\
\hline \multicolumn{4}{|c|}{ Año 2013} \\
\hline Carne de ave (pollo) & Cajamarca y Arequipa & Arequipa y San Mart $n$ & 0 \\
\hline Carne de bovino & 0 & 0 & 0 \\
\hline Carne de ovino & 0 & Cajamarca & 0 \\
\hline Carne de caprino & 0 & 0 & 0 \\
\hline Carne de porcino & 0 & $\begin{array}{l}\text { Ica, La Libertad, Lima Callao, } \\
\text { Tacna y Piura }\end{array}$ & La Libertad \\
\hline Carne de cuy & Lima Callao & Puno y Lima Callao & Lima Callao \\
\hline Leche cruda de bovino & 0 & San Mart h́ & 0 \\
\hline Alpaca/llama & 0 & 0 & 0 \\
\hline Miel de abeja & Ica & 0 & Tacna e Ica \\
\hline \multicolumn{4}{|c|}{ Año 2014} \\
\hline Carne de ave (pollo) & Tacna & Tacna & 0 \\
\hline Carne de bovino & La Libertad & La Libertad y Puno & 0 \\
\hline Carne de ovino & 0 & 0 & 0 \\
\hline Carne de caprino & 0 & 0 & 0 \\
\hline Carne de porcino & Ica & 0 & 0 \\
\hline Carne de cuy & La Libertad & La Libertad y Arequipa & 0 \\
\hline Leche cruda de bovino & Arequipa & 0 & 0 \\
\hline Alpaca/llama & 0 & 0 & 0 \\
\hline Miel de abeja & Ica, Tacna & 0 & Piura \\
\hline \multicolumn{4}{|c|}{ Año 2015} \\
\hline Carne de ave (pollo) & 0 & 0 & 0 \\
\hline Carne de bovino & 0 & 0 & 0 \\
\hline Carne de ovino & 0 & 0 & 0 \\
\hline Carne de caprino & 0 & 0 & 0 \\
\hline Carne de porcino & 0 & 0 & 0 \\
\hline Carne de cuy & Arequipa & 0 & 0 \\
\hline Leche cruda de bovino & Arequipa & Arequipa & 0 \\
\hline Alpaca/llama & 0 & 0 & 0 \\
\hline Miel de abeja & 0 & 0 & 0 \\
\hline \multicolumn{4}{|c|}{ Año 2016} \\
\hline Carne de ave (pollo) & 0 & 0 & 0 \\
\hline Carne de bovino & 0 & 0 & 0 \\
\hline Carne de ovino & 0 & 0 & 0 \\
\hline Carne de caprino & 0 & 0 & 0 \\
\hline Carne de porcino & 0 & 0 & 0 \\
\hline Carne de cuy & 0 & 0 & 0 \\
\hline Leche cruda de bovino & 0 & 0 & 0 \\
\hline Alpaca/llama & 0 & 0 & 0 \\
\hline Miel de abeja & 0 & 0 & 0 \\
\hline \multicolumn{4}{|c|}{ Año 2017} \\
\hline Carne de ave (pollo) & 0 & 0 & 0 \\
\hline Carne de bovino & 0 & 0 & 0 \\
\hline Carne de ovino & 0 & 0 & 0 \\
\hline Carne de caprino & 0 & 0 & 0 \\
\hline Carne de porcino & 0 & 0 & 0 \\
\hline Carne de cuy & Cajamarca & 0 & 0 \\
\hline Leche cruda de bovino & 0 & 0 & 0 \\
\hline Alpaca/llama & 0 & 0 & Puno \\
\hline Miel de abeja & 0 & 0 & 0 \\
\hline \multicolumn{4}{|c|}{ Año 2018} \\
\hline Carne de ave (pollo) & San Mart ńn & 0 & 0 \\
\hline Carne de bovino & 0 & 0 & 0 \\
\hline Carne de ovino & La Libertad & 0 & 0 \\
\hline Carne de caprino & 0 & 0 & 0 \\
\hline Carne de porcino & 0 & 0 & 0 \\
\hline Carne de cuy & 0 & Lambayeque & 0 \\
\hline Leche cruda de bovino & 0 & Ica, Lambayeque & 0 \\
\hline Alpaca/llama & 0 & 0 & 0 \\
\hline
\end{tabular}

Ciencia Latina Revista Científica Multidisciplinar, Ciudad de México, México. ISSN 2707-2207 / ISSN 2707-2215 (en línea), marzo-abril, 2021, Volumen 5, Número 2. https://doi.org/10.37811/cl rcm.v5i2.418 p. 2073 
La presencia de residuos de antibióticos fue reportada en todo el período (2011-2018), con excepción del año 2016, siendo el número de localidades reportadas mayor en el 2014 y menor en el 2017. El residuo AMOZ, de la furaltadona, se reportó en 9 regiones del país, el residuo AOZ, de la furazolidona, se reportó en 11 regiones del país, en tanto que el CAP fue reportado en 7 regiones. Las regiones que reportaron la presencia de estos 3 antibióticos o sus residuos, en el tiempo de evaluación (2011 - 2018) fueron: La Libertad, Lima, Callao, Ica, y Tacna, lo que evidencia potencial riesgo para la salud humana de estos xenobióticos en todo el período de evaluación. San Martín, La Libertad, Lambayeque e Ica, fueron las regiones donde el uso de estos xenobióticos fue más reciente, dentro del período de estudio (Tabla 1).

Los nitrofuranos son antibióticos sintéticos de amplio espectro que han sido reportados en alimentos de origen animal, incluyendo leche y productos lácteos (EFSA Panel on Contaminants in the Food Chain (CONTAM), 2015), y sus metabolitos derivados han mostrado efectos cancerígenos y mutagénicos (Yahagi et al., 1974; McCalla, 1977), de ahí que se haya dispuesto su prohibición en la década de los '90 en los Estados Unidos (Radovnikovic et al., 2011) así como en la Unión Europea, Tailandia, Australia, Filipinas y Brasil (Islam et al., 2014). En el Perú se prohibieron desde el 2013 (SENASA, 2013), sin embargo se considera que corresponde el monitoreo de estos xenobióticos o sus metabolitos a una política coherente de seguridad alimentaria. Dentro de los nitrofuranos se tiene originalmente a la furaltadona, furazolidona, nitrofurantoína y nitrofurazona, que actúan como antibióticos de amplio espectro que se metabolizan rápidamente a metabolitos que se asocian a proteínas, siendo relevante la determinación de trazas de sus metabolitos por ser más estables en el organismo (Yan et al., 2020) incluso varias semanas después de su administración, de ahí que, como residuos marcadores se tenga a la 3-amino-5-morfolino-metil-1,3-oxa-zolidinona (AMOZ, el residuo marcador de furaltadona) y la 3-amino-2-ozaxolidinona (AOZ, el residuo marcador de furazolidona) (Martos \& Shurmer, 2012). 
Tabla 2: Presencia de residuos de furaltadona (AMOZ) en alimentos de procesamiento primario (2011-2018)

\begin{tabular}{|c|c|c|c|c|c|c|c|c|c|}
\hline \multirow{3}{*}{ Matriz } & \multicolumn{8}{|c|}{ FURALTADONA (AMOZ) } & \multirow[b]{3}{*}{ San Martín } \\
\hline & \multicolumn{8}{|c|}{ Región } & \\
\hline & Lambayeque & La Libertad & Lima & Callao & Ica & Arequipa & Tacna & Cajamarca & \\
\hline Carne de ave (pollo) & 2011 & - & 2011 & - & - & 2013 & 2014 & 2013 & 2018 \\
\hline Carne de bovino & - & 2014 & - & - & - & - & - & - & \\
\hline Carne de ovino & - & 2012; 2018 & - & - & - & - & - & - & \\
\hline Carne de caprino & - & - & - & - & - & - & - & - & \\
\hline Carne de porcino & - & - & - & - & 2014 & - & 2011 & 2011 & \\
\hline Carne de cuy & - & 2014 & 2013 & 2013 & - & 2015 & - & 2017 & \\
\hline Leche cruda de bovino & - & - & - & - & - & $2014 ; 2015$ & - & - & \\
\hline Alpaca/llama & - & - & - & - & - & - & - & - & \\
\hline Miel de abeja & - & - & - & - & $2013 ; 2014$ & - & 2014 & - & \\
\hline
\end{tabular}

La Libertad, Arequipa, Tacna, Cajamarca, Ica, Lambayeque, Lima y San Martín fueron las regiones donde el reporte fue más frecuente tanto para AMOZ como para AOZ (Tablas 2 y 3), sin embargo a nivel de regiones existió un mayor uso de furazolidona (11 regiones) que de furaltadona (9 regiones), probablemente al ser usadas en las raciones de alimentos de aves de corral por su efecto coccidiostático y para disminuir la muerte a causa de sepsis por bacterias Gram-negativas (Russell, 2014). La AMOZ fue reportada en la producción de aves de corral (seis regiones); crianza de cuyes (cinco regiones); producción de carne de porcino (tres regiones); producción de miel de abeja (dos regiones, incluyendo Ica en dos años consecutivos); y producción de carne de bovino, producción de carne de ovino, y producción de leche cruda de bovino (región Arequipa, en dos años consecutivos) (Tabla 2).

Tabla 3: Presencia de residuos de furazolidona (AOZ) en alimentos de procesamiento primario (2011-2018)

\begin{tabular}{|c|c|c|c|c|c|c|c|c|c|c|c|}
\hline \multirow{3}{*}{ Matriz } & \multirow{2}{*}{\multicolumn{11}{|c|}{$\begin{array}{l}\text { FURAZOLIDONA (AOZ) } \\
\text { Región y año de reporte }\end{array}$}} \\
\hline & & & & & & & & & & & \\
\hline & Piura & Lambayeque & La Libertad & Lima & Callao & Ica & Arequipa & Tacna & Cajamarca & Puno & San Martín \\
\hline Carne de ave (pollo) & - & 2011 & 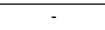 & 2011 & - & - & 2013 & 2012; 2014 & & - & $2012 ; 2013$ \\
\hline Carne de bovino & - & - & 2014 & - & - & - & - & - & - & 2014 & - \\
\hline Carne de ovino & - & - & 2012 & - & - & - & - & - & 2013 & - & - \\
\hline Carne de caprino & - & - & - & - & - & - & - & - & - & - & - \\
\hline Carne de porcino & 2012; 2013 & - & 2013 & 2013 & 2013 & 2013 & - & 2011; 2012; 2013 & 2011 & - & - \\
\hline Carne de cuy & - & 2018 & 2014 & 2013 & 2013 & - & 2014 & - & - & 2013 & - \\
\hline Leche cruda de bovino & - & 2018 & - & - & - & 2018 & 2015 & - & - & - & 2013 \\
\hline Alpaca/llama & - & - & - & - & - & - & - & - & - & - & - \\
\hline Miel de abeja & - & - & - & - & - & - & - & - & - & - & - \\
\hline
\end{tabular}

La AOZ fue detectada en carne de porcino (siete regiones), reportándose en Tacna en tres años consecutivos (2011-2013) y en Piura en dos años consecutivos (2012-2013); carne de cuy, (seis regiones); carne de aves de corral (cinco regiones), reportándose en San Martín, en dos años consecutivos (2012 y 2013); leche cruda de bovino (cuatro regiones); carne de bovino (dos regiones); y carne de ovino (dos regiones). A su vez se pudo 
evidenciar que las regiones de Lambayeque e Ica fueron las mas recientes en el uso de este xenobiótico dentro del período de estudio (Tabla 3).

Tabla 4: Presencia de cloranfenicol (CAP) en alimentos de procesamiento primario (2011-2018)

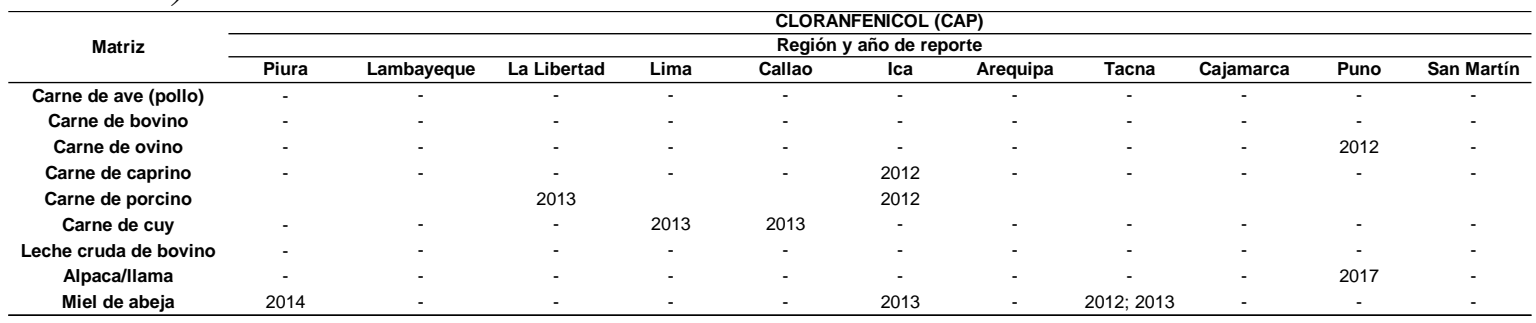

El cloranfenicol (CAP), por su bajo costo y alta eficacia para tratar enfermedades como la gastroenteritis bacteriana, ha sido utilizado más allá del período de retiro durante la lactancia en vacunos, lo que ha conllevado a que se reporte en tejido animal y leche cruda (Xie et al., 2017). En humanos puede causar una enfermedad grave que puede conllevar a la muerte, la anemia aplásica, independientemente de la cantidad ingerida así como la suspensión de la ingesta alimentaria de la fuente que lo contiene (Polyanskikh et al., 2019) siendo prohibido en animales productores de alimentos en países como Estados Unidos y Australia (Page, 1991) no habiendo conciencia de su uso en la producción avícola y ganadera (Ezenduka et al., 2018).

Para el CAP, a diferencia de la AMOZ y la AOZ (reportados hasta el 2018), sólo hay reporte hasta el 2017. Además, su mayor uso fue en la producción de miel de abeja en tres regiones, reportándose en Ica hasta en dos años consecutivos (2012 y 2013); carne de cuy, en dos regiones; carne de porcino, en dos regiones; carne de caprino, en una región; carne de ovino, en una región; y carne de camélido en una región (Tablas 1 y 4). A su vez se puede evidenciar que Puno fue la región donde el uso de este xenobiótico fue más reciente dentro del período de estudio.

Dado que los nitrofuranos y el cloranfenicol son los únicos antibióticos prohibidos para la producción de animales para consumo humano (Kirchhelle, 2018), incluyendo el Perú (SENASA, 2013), es preocupante que se reporten estos xenobióticos en diferentes matrices animales hasta cinco años después de la prohibición y en la tres regiones del país por lo que amerita reforzar y ampliar el monitoreo de estos antibióticos en todo el país, además de efectuar una vigilancia de casos de efectos adversos para la salud humana por 
el consumo de alimentos animales en aquellas localidades donde se haya reportado su presencia.

\section{CONCLUSIÓN O CONSIDERACIONES FINALES}

En conclusión se puede señalar que tanto los antibióticos prohibidos cloranfenicol y los nitrofuranos furaltadona y furazolidona han seguido usándose más allá del período de su prohibición y en algunas regiones su uso ha sido más reciente y más frecuente (a través de los años) y en más matrices alimentarias, por lo que se corresponde continuar con el monitoreo y a la vez transparentar los hallazgos encontrados a efectos de prevenir potenciales efectos para la salud humana.

\section{LISTA DE REFERENCIAS}

Abdelsalam, N., Ramadan, A., ElRakaiby, M., \& Aziz, R. (2020). Toxicomicrobiomics: The Human Microbiome vs. Pharmaceutical, Dietary, and Environmental Xenobiotics. Frontiers In Pharmacology, 11. https://doi.org/10.3389/fphar.2020.00390

Beyene, T., \& Assefa, S. (2015). Assessment of Rational Veterinary Drugs Use in Livestock at Adama District Veterinary Clinic, Central Ethiopia. Journal of Veterinary Science \& Technology,07(03). https://doi.org/10.4172/21577579.1000319

Boyd, W. (2001). Making Meat: Science, Technology, and American Poultry Production. Technology And Culture, 42(4), 631-664. https://doi.org/10.1353/tech.2001.0150

Briceño-Fereira, E. del C., Ascanio-Evanoff, E. R., Riera- Betancourt, J. G., ArrietaMendoza, D., Flores-Chona, S. A., \& Maniglia-Mérida, G. C. (2010). Evidencia de residuos de cloranfenicol en muestras de riñón de cerdos de la región Central de Venezuela. Revista Científica, 20(3), 254-258.

Colombo, R., \& Papetti, A. (2019). Advances in the Analysis of Veterinary Drug Residues in Food Matrices by Capillary Electrophoresis Techniques. Molecules, 24(24). https://doi.org/10.3390/molecules24244617

EFSA Panel on Contaminants in the Food Chain (CONTAM). (2015). Scientific Opinion on nitrofurans and their metabolites in food: Nitrofurans in food. EFSA Journal, 13(6), 4140 . 
Ezenduka, E., Okonkwo, B., Anyaoha, C., Nwanta, J., \& Anaga, A. (2018). Immunologic determination of chloramphenicol residue in commercial birds at Nsukka, Enugu State, Southeast Nigeria. International Journal Of One Health, 4, 22-27. https://doi.org/10.14202/ijoh.2018.22-27

FAO-OMS. (2018). Límites máximos de residuos (LMR) y recomendaciones sobre la gestión de riesgos (RGR) para residuos de medicamentos veterinarios en los alimentos $C X / M R L$ 2-2018 [Ebook] (p. 45). Retrieved 7 February 2021, from http://www.fao.org/fao-who-codexalimentarius/shproxy/en/?lnk=1\&url=https $\% 253 \mathrm{~A} \% 252 \mathrm{~F} \% 252 \mathrm{Fworkspace}$.fao.org\% $\% 252 \mathrm{Fsites} \%$ 252Fcodex\%252FStandards\%252FCXM\%2B2\%252FMRL2s.pdfCode.

FDA. (2013). 2013 SUMMARY REPORT On Antimicrobials Sold or Distributed for Use in Food-Producing Animals. https://www.fda.gov/media/91700/download

Islam, M. J., Lisa, A. A., Reza, A. H. M. M., Reza, M. S., Absar Khan, M. N., \& Kamal, M. (2014). Source identification and entry pathways of banned antibiotics nitrofuran and chloramphenicol in shrimp value chain of Bangladesh. Eurasian Journal of Biosciences, 71-83.

Kirchhelle, C. (2018). Pharming animals: a global history of antibiotics in food production (1935-2017). In Palgrave Communications (Vol. 4, Issue 1). https://doi.org/10.1057/s41599-018-0152-2

Martos, P., \& Shurmer, B. (2012). Sample Preparation Techniques for the Determination of Veterinary Drugs in Food Matrices. Comprehensive Sampling And Sample Preparation, 405-414. doi: 10.1016/b978-0-12-381373-2.00141-1

McCalla, D. R. (1977). Biological effects of nitrofurans. The Journal of Antimicrobial Chemotherapy, 3(5), 517-520.

MINSA. (2016). RM-372-2016-MINSA-con-NTS-120-MINSA-DIGESA-v01-LMRMedicamentos.pdf. https://www.senasa.gob.pe/senasa/descargasarchivos/2014/11/RM-372-2016MINSA-con-NTS-120-MINSA-DIGESA-v01-LMR-Medicamentos.pdf.

Page, S. W. (1991). Chloramphenicol 1. Hazards of use and the current regulatory environment. Australian Veterinary Journal, 68(1), 1-2.

Polyanskikh, E. I., Polonevich, A. G., Belysheva, L. L., Rakhman'ko, E. M., \& Leshchev, S. M. (2019). A Procedure for the Control of the Residual Chloramphenicol 
(Laevomycetin) in Food Products of Animal Origin. Journal of Analytical Chemistry, 74(6), 601-608.

Radovnikovic, A., Moloney, M., Byrne, P., \& Danaher, M. (2011). Detection of banned nitrofuran metabolites in animal plasma samples using UHPLC-MS/MS. Journal of Chromatography. B, Analytical Technologies in the Biomedical and Life Sciences, 879(2), 159-166.

Russell, D. (2014). Comparative Pathology: Cardiovascular System. Pathobiology of Human Disease, 1042-1070. https://doi.org/10.1016/b978-0-12-386456-7.03403-1

SENASA (2013). Resolución Directoral No0072-2013-MINAGRI-SENASA-DIAIA. Retrieved January 29, 2021, from Gob.pe website: https://www.minagri.gob.pe/portal/resoluciones-directorales/rd-2013/9631resolucion-directoral-n-0072-2013-minagri-senasa-diaia

SENASA (2017). Retrieved 7 February 2021, from https://www.senasa.gob.pe/senasa/descargasarchivos/2016/08/Informe-demonitoreo-2016.pdf.

SENASA (2020). Programa Nacional de Monitoreo de Contaminantes. https://www.senasa.gob.pe/senasa/programa-nacional-de-monitoreo/

USDA (2015) UNITED STATES National Residue Program for Meat, Poultry, and Egg Products $2015 \quad$ Residue Sampling Plans. https://www.fsis.usda.gov/wps/wcm/connect/04c818ed-9bb1-44b2-9e3f896461f1 ffb9/2015-Blue-Book.pdf?MOD=AJPERES

Xie, Y., Hu, Q., Zhao, M., Cheng, Y., Guo, Y., Qian, H., \& Yao, W. (2017). Simultaneous Determination of Erythromycin, Tetracycline, and Chloramphenicol Residue in Raw Milk by Molecularly Imprinted Polymer Mixed with Solid-Phase Extraction. Food Analytical Methods, 11(2), 374-381. https://doi.org/10.1007/s12161-017-1008-X

Yahagi, T., Nagao, M., HarÃ, K., Matsushima, T., Sugimura, T., \& Bryan, G. T. (1974). Relationships between the Carcinogenic and Mutagenic or DNA-modifying Effects of Nitrofuran Derivatives, Including 2-(2-Furyl)-3-(5-nitro-2-furyl)acrylamide, a Food Additive1. Cancer Research, 34, 2266-2273.

Yan, C., Teng, J., Liu, F., Yao, B., Xu, Z., Yao, L., \& Chen, W. (2020). Signal amplified enzyme-linked immunosorbent assay with gold nanoparticles for sensitive 
detection of trace furaltadone metabolite. Microchemical Journal, 159, 105414. doi: 10.1016/j.microc.2020.105414 